\title{
«Eine Brücke schaffen \\ von der Medizin zum Bürger»
}

\section{Adrian Ritter}

Freier Journalist

Vor 30 Jahren begann Silva Keberle neben ihrer Tätigkeit als Assistenzärztin im Kantonsspital Basel, die Öffentlichkeit über medizinische Themen zu informieren. Ein Blick auf die Geschichte ihrer Firma Eskamed AG und die Entwicklung der Gesundheitskommunikation.

Der Aufruhr war gross, als Silva Keberle am nächsten Tag im Kantonsspital Basel zur Arbeit erschien. Die 32-jährige Assistenzärztin hatte am Tag zuvor auf Radio Basilisk eine Sendung zum Thema "Aids» moderiert. Es war das Jahr 1987, die Krankheit neu und vielen noch unbekannt. Silva Keberle war seit einigen Jahren als «Radioärztin» tätig und informierte die Hörerinnen und Hörer über Krankheiten, deren Ursachen und Behandlungsmöglichkeiten. Sie betreibe Panikmache, rügte sie ihr Vorgesetzter am Kantonsspital. Verunsicherte Zuhörer seien nach der Sendung zu einem HIVTest ins Spital gekommen. Panikmache? Das Gegenteil war die Absicht von Silva Keberle gewesen: ruhig und sachlich über medizinisch relevante Themen informieren. Dass die Aids-Sendung berechtigt war, zeigte sich, als nicht wenige der Verunsicherten sich beim Test als HIV-positiv erwiesen.

Es waren ihre Erfahrungen als Assistenzärztin im Spital gewesen, die Silva Keberle auf die Idee mit der Öffentlichkeitsarbeit gebracht hatten. Die Ärzte haben zu wenig Zeit und nicht die nötige Schulung, um den Patienten die nötigen Informationen verständlich und geduldig zu vermitteln, hatte sie festgestellt. «Ärzte können nicht Volkshochschule betreiben am Spitalbett, das ist klar», sagt die heute 62-Jährige rückblickend: «Es ging mir damals darum, überhaupt ein Be-

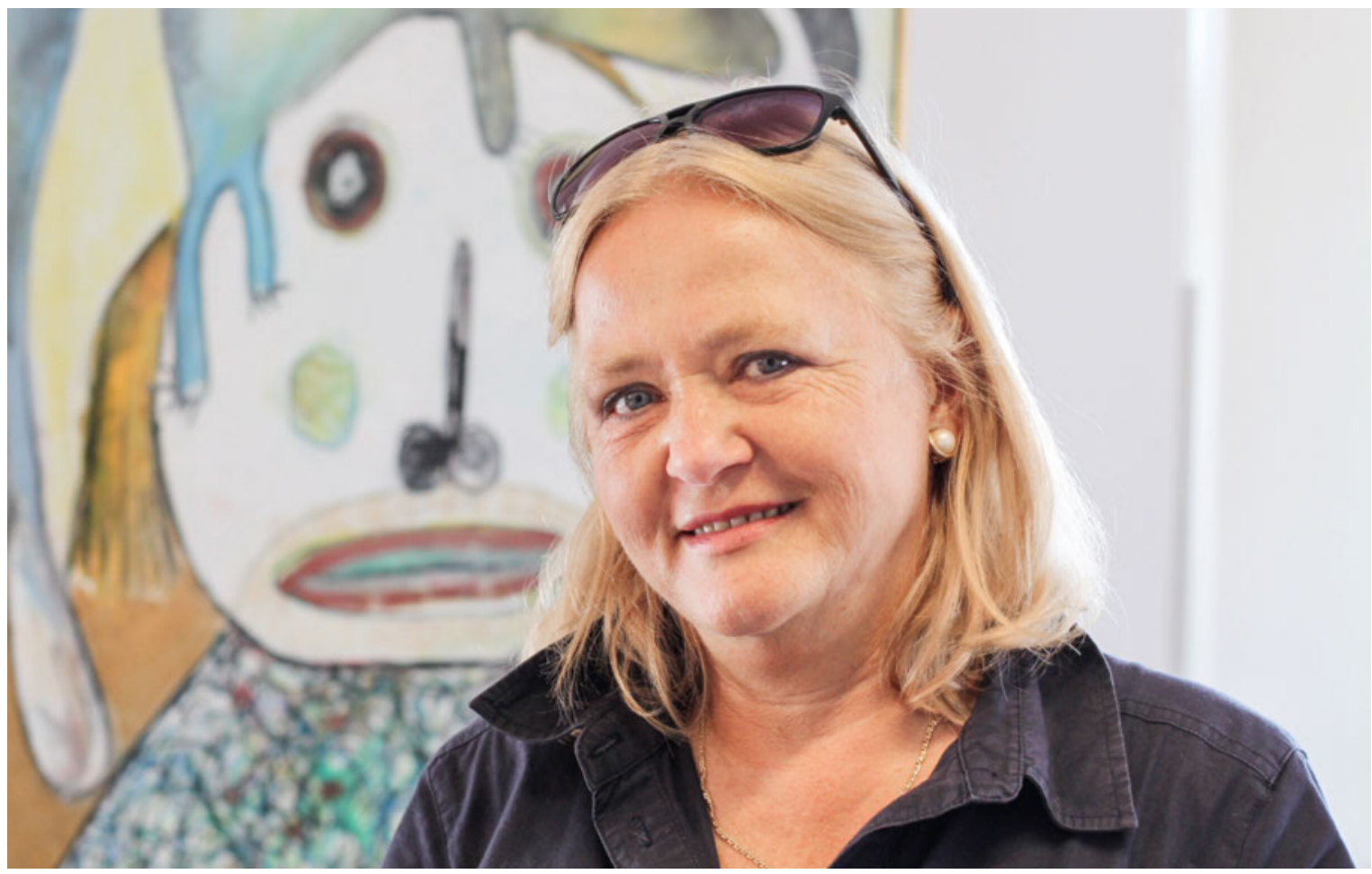

«Mein Grundanliegen ist der Schutz der Patientinnen und Patienten»: Eskamed-Gründerin Silva Keberle. (Foto: Adrian Ritter) 


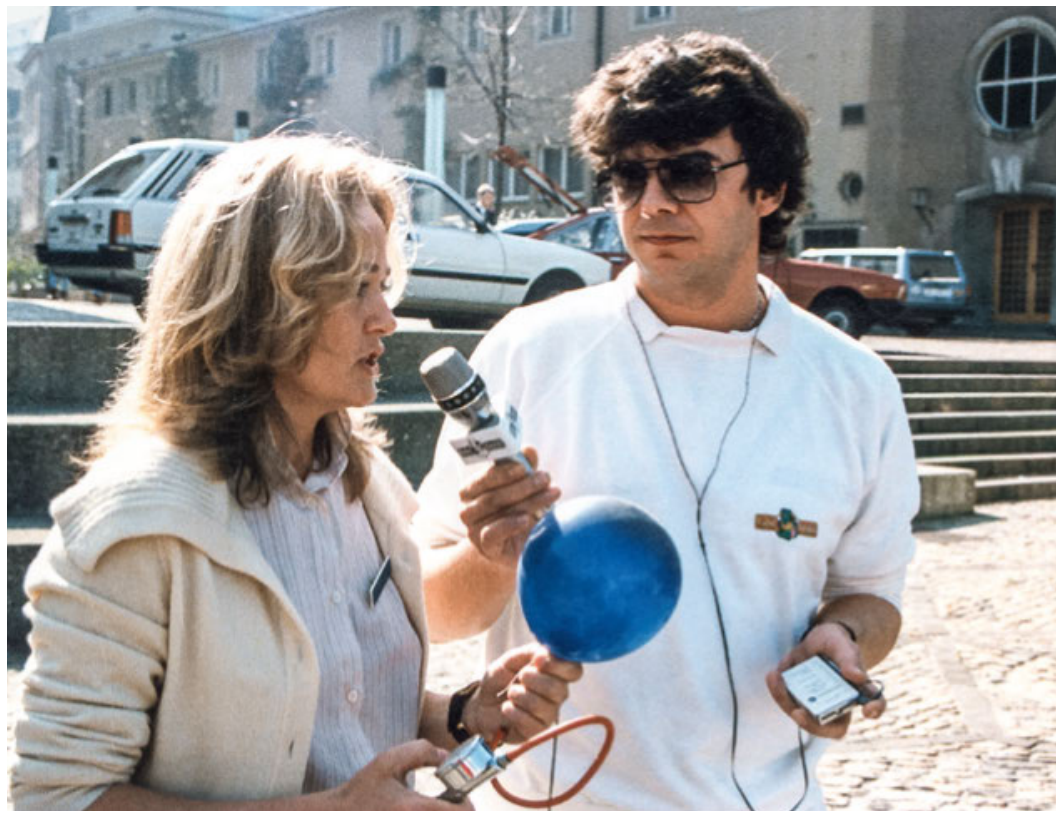

Aktion «Blutdruck messen nicht vergessen»: Silva Keberle bietet 1986 mit Radio Basilisk auf dem Barfüsserplatz in Basel öffentliches Blutdruckmessen an.

(Foto: $z \mathrm{Vg}$ von Silva Keberle)

wusstsein für Gesundheit zu schaffen und eine Brücke vom Wissen der Medizin zum Bürger zu bauen. Mein Ziel war es, verständlich und ohne Drohfinger den Patienten ein Grundwissen zu medizinischen Themen zu vermitteln. Damit sie den Mut haben, die für ihre konkrete Situation richtigen Fragen zu stellen und die Erklärungen des Arztes zu verstehen», so Keberle.

\section{Der Schritt in die Selbständigkeit}

Mit diesem Ziel hatte Silva Keberle begonnen, neben ihrer Assistenzstelle über medizinische Themen zu schreiben, etwa für die damalige Basler NationalZeitung und die Coop-Zeitung. Von 1983 bis 1993 moderierte sie Sendungen auf Radio Basilisk. «Ich war vermutlich die erste Ärztin in der Schweiz, die regelmässig in den Medien präsent war», so Keberle.

Schliesslich gab sie ihre Tätigkeit als Ärztin auf, widmete sich ganz der Gesundheitskommunikation und gründete dazu 1986 die Eskamed AG. Lehrmittel, Lexika, die Zeitschrift Therapiewoche und Videos: Die Firma entwickelte eine breite Palette an Produkten. Wichtig war Silva Keberle immer auch das Spielerische. So entstanden auch Kartenspiele, die Kinder über gesundheitliche Gefahren informieren. Besonders erfolgreich war das 1998 erschienene Buch Meine Gesundheit, ein Ratgeber für die ganze Familie.

Für erneute Furore in Ärztekreisen sorgte Silva Keberle mit einer Innovation, die 1990 auf die Schweizer Strassen rollte: Der rote Denner-Check-up-Bus mit Krankenpflegepersonal an Bord bot Blutdruck- und Cho- lesterinmessungen an. Einige Ärzte kritisierten die «Konkurrenz». Keberle konnte im Gespräch die Wogen glätten: «Es ging ja genau darum, dass Menschen mit zu hohen Blutdruckwerten ihren Hausarzt aufsuchen, um sich weiter abklären zu lassen", sagt sie im Rückblick. Tempi passati: Heute kann man sich in jeder Apotheke und auf jeder Gewerbeschau den Blutdruck messen lassen, ohne dass dies eine Aufregung auslöst.

\section{Die neue Unübersichtlichkeit}

Auch sonst hat sich die Gesundheitskommunikation in den letzten 30 Jahren stark verändert, stellt Keberle fest: «Früher erwarteten die Patienten, dass der Arzt allwissend ist. Heute will sich der Bürger selber informieren und als mündiger, selbstverantwortlicher Patient in konstruktiver Zusammenarbeit mit dem Arzt Entscheidungen treffen.»

An Informationen mangle es heute nicht mehr. Manch ein chronischer Patient sei besser über seine Krankheit informiert als sein Hausarzt. Gleichzeitig sei mit der Informationsflut im Internet aber die Verunsicherung in der Bevölkerung gestiegen. Die Medien tragen gemäss Keberle das ihre dazu bei, indem sie vor allem auf ihre Sponsoren und Einschaltquoten schielen und bei der Jagd nach Primeuren bisweilen unnötig Angst verbreiten. Das Resultat: «In der Bevölkerung herrscht zu medizinischen Themen nicht selten ein gefährliches Halbwissen.» Der Bedarf an fachlich fundierten Informationsmitteln zu medizinischen Themen sei deshalb heute nicht kleiner.

\section{Eine Wahl ermöglichen}

Keberle geht es dabei vor allem um den Schutz der Patientinnen und Patienten. Nicht bevormunden wolle sie diese, sondern Wahlmöglichkeiten im Dickicht der modernen Medizin schaffen. Diesem Ziel dient auch ein Projekt, das die Eskamed 1999 startete - das ErfahrungsMedizinische Register (EMR). Dort sind heute rund 20000 Therapeutinnen und Therapeuten der Komplementärmedizin registriert und das EMR-Qualitätslabel wird von über 40 Krankenversicherungen genutzt. «Das war Pionierarbeit. Es gab zu Beginn keine einheitliche Definition, was Komplementärmedizin überhaupt umfasst, und keinen Konsens, welche Therapien vertrauenswürdig sind. Wir mussten erst entsprechende Qualitätsmassstäbe entwickeln.» Inzwischen entwickelt Eskamed auch für Österreich ein solches Register und ein entsprechendes Projekt in Deutschland ist in Diskussion.

Neben dem ErfahrungsMedizinischen Register hat die Firma von Silva Keberle in den letzten Jahren ein Regis- 


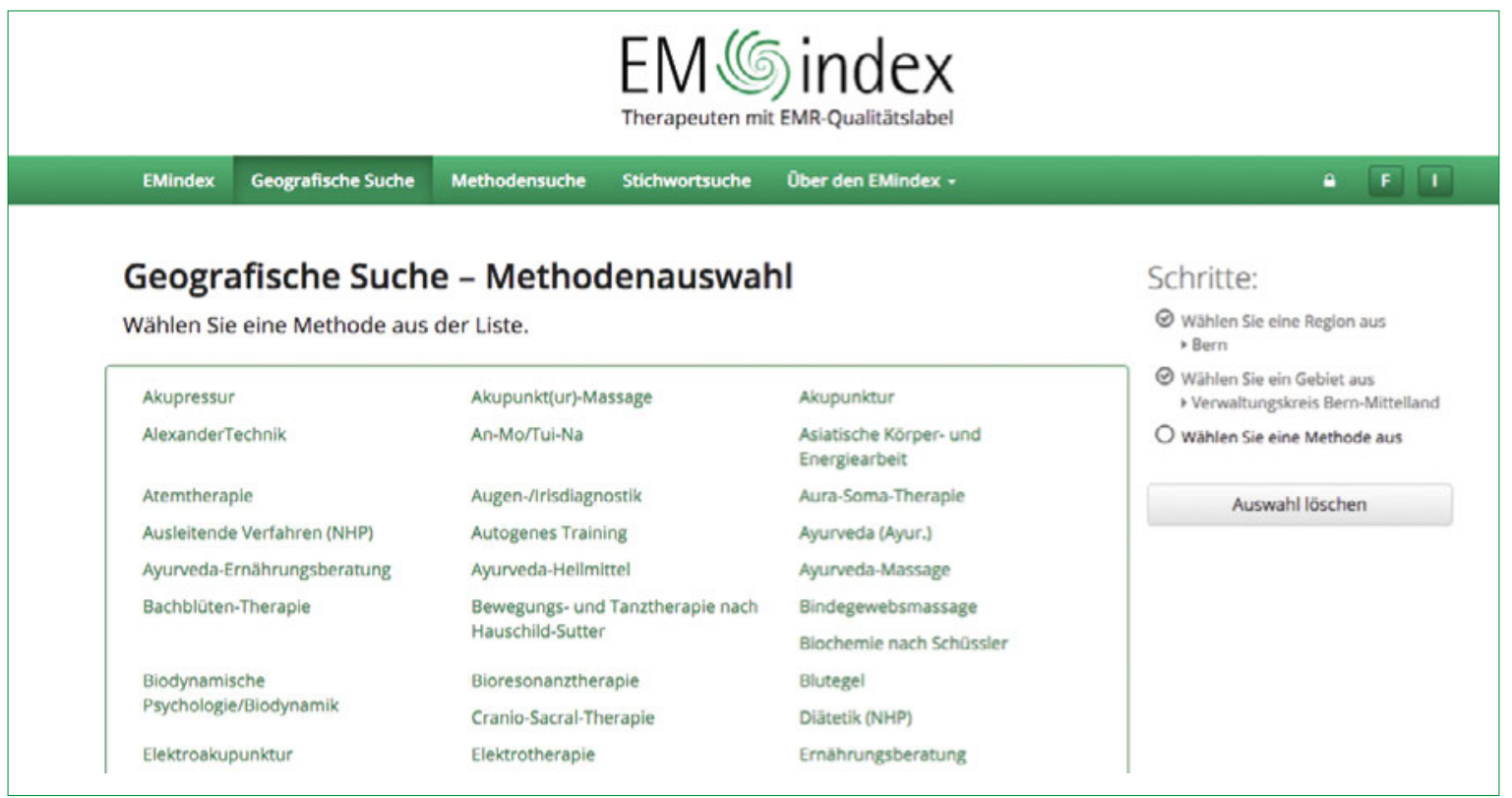

Gesundheitsinformation heute: Im ErfahrungsMedizinischen Register (EMR) der Eskamed AG sind rund 20000 Therapeutinnen und Therapeuten der Komplementärmedizin registriert. (Foto: Screenshot von der Homepage)

ter für den immer wichtigeren Bereich der Gesundheitsförderung aufgebaut. EMfit ist ein Qualitätslabel für Kursleitende - von Angeboten wie Aquafit bis Yoga. Weil diese Projekte die Arbeit der Eskamed stark prägten, gründete Silva Keberle 2002 zusätzlich die Eskamedia AG, in welcher seither die Projekte der Gesundheitskommunikation angesiedelt sind. Beide Firmen haben ihren Sitz in Basel und beschäftigen heute insgesamt rund 50 Mitarbeitende.

\section{Heute fehlt die Zeit}

Auch wenn Silva Keberle schon lange nicht mehr als praktische Ärztin tätig ist, ist sie dem Berufsstand doch verbunden geblieben. «Die anfängliche Angst vor Konkurrenz und der Vorwurf der Panikmache ist einer konstruktiven Zusammenarbeit gewichen», erzählt sie lachend. So war sie 1991 bis 2009 Vorstandsmitglied der Schweizerischen Gesellschaft für Innere Medizin (SGIM) und leitete viele Jahre auch deren Geschäftsstelle. Seit 2008 ist Keberle Mitglied und seit 2012 Präsidentin der Eidgenössischen Kommission für allgemeine Leistungen und Grundsatzfragen (ELGK).

Wie beurteilt sie heute die Fähigkeit der Ärztinnen und Ärzte, ihre Patienten verständlich und geduldig zu munikation mit dem Patienten für den Therapieerfolg sei längst erkannt und werde inzwischen in Studium und Weiterbildungen geschult. Um das Gespräch zwischen Arzt und Patient sei es heute aus anderen Gründen oft nicht besser gestellt als früher: «Der Effizienzdruck im Gesundheitswesen lässt schlicht zu wenig Zeit dafür.» Umso mehr interessiert sich Silva Keberle für die politischen und ethischen Aspekte, welche die Arbeitsbedingungen der Ärztinnen und Ärzte massgeblich mitbestimmen.

Es brauche zweierlei, ist Keberle überzeugt: sinnvolle Rahmenbedingungen, die den Ärztinnen und Ärzten Zeit lassen für das Gespräch mit dem Patienten. Weil Ärzte aber auch heute nicht Volkshochschule betreiben könnten, seien nach wie vor qualitativ hochstehende Produkte der Gesundheitskommunikation gefragt. Ein solches Produkt, das sie gerne noch entwickeln möchte, ist ein Medium, das auf positive Nachrichten aus Gesundheit und Medizin fokussiert - fern von Panikmache und nah beim Vertrauen auf die Medizin und die Selbstheilungskräfte in jedem Menschen.

\section{Weiterführende Informationen}

15 Jahre EMR: Interview mit Silva Keberle: Schweiz Ärztezeitung. 2014;95:38, www.saez.ch/docs/saez/2014/38/de/saez-02972.pdf 